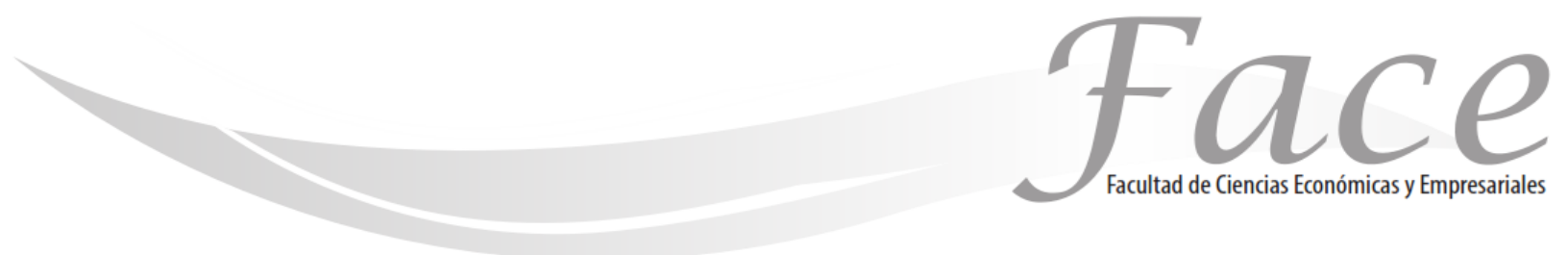

ISSN Impreso: 1794-9920

ISSN Electrónico: 2500-9338

Volumen $18-\mathrm{N}^{\circ} 1$

Año 2018

Págs. 22 - 32

\title{
LA PUBLICIDAD EN REDES SOCIALES COMO UNA ALTERNATIVA SOSTENIBLE PARA EL DESTINO TURÍSTICO BAHÍA DE CARÁQUEZ - ECUADOR
}

\author{
Frank Ángel Lemoine Quintero* \\ Enlace ORCID: https://orcid.org/0000-0001-8885-8498 \\ Gema Lourdes Lopéz Zambrano** \\ Enlace ORCID: https://orcid.org/0000-0003-4559-3885 \\ Wiffredo Valls Figueroa *** \\ Enlace ORCID: https://orcid.org/0000-0002-1194-1020
}

Fecha de Recepción: 1 de Mayo 2018

Fecha de Aprobación: 31 de Julio 2018

\section{Resumen:}

En la presente investigación se analizó la publicidad en la red social de Facebook y su impacto en el desarrollo turístico, caso H Sports Café Bahía de Caráquez. La información obtenida a través del campo bibliográfico y la red social permitió realizar un análisis descriptivo mediante tablas de datos en excel con la información de las publicaciones y anuncios, tomando en cuenta los parámetros de: visualizaciones, likes, comentarios y número de veces compartido de los contenidos.Se comprobó que las visualizaciones de los anuncios publicados en la Fanpage amentaron de 44639 en marzo del 2016 a 51362 en enero del 2017. Se midió el impacto de los anuncios por familia de productos representando los cocteles el $40,10 \%(\$ 39629,96)$ del total de las ventas, las cervezas artesanales el $25,31 \%(\$ 25010,43)$, las alitas, nachos y Nuggets el $9,35 \%$, (\$9239,64) y los desayunos el $8,65 \%(\$ 8549,45)$ constatando que las ventas del negocio han tenido un aumento significativo. Por ende se demuestra que la publicidad en la red social de Facebook si contribuye al desarrollo del turismo y crecimiento económico de los negocios.

Palabras Clave: Publicidad, Desarrollo turístico, Turismo, Red Social, Ventas.

\footnotetext{
* Docente titular de la Universidad Laica Eloy Alfaro de Manabí, Extensión Bahía de Caráquez. Master en Administración de Empresas .Investigador acreditado por la SENESCYT reg-inv-16-01382 y líder del proyecto de vinculación con la sociedad de Rediseño de Imagen de los pequeños negocios de Leónidas Plaza, Manabí, Ecuador. Correo Electrónico: fangel64@gmail.com

**. Ingeniera en marketing de la Universidad Laica Eloy Alfaro de Manabí, Extensión Bahía de Caráquez - Ecuador. Miembro del grupo científico estudiantil de la Extensión. Correo Electrónico: gemaposli@hotmail.com

*** Ingeniero Industrial de la Universidad de Matanzas, Cuba. Docente tiempo completo de la carrera de Hotelería y Turismo de la Extensión Bahía de Caráquez de la Universidad laica Eloy Alfaro de Manabí. Presidente de la comisión de Investigación y líder del proyecto institucional Desarrollo del turismo sostenible en destino Sucre, San Vicente, Jama y Pedernales. Máster en Gestión de Empresas Turísticas de la Universidad de Palma. Gran Canaria y doctor en Ciencias Técnica. Correo Electrónico: wilfredovalls1957@gmail.com
} 


\title{
ADVERTISING IN SOCIAL NETWORKS AS A SUSTAINABLE ALTERNATIVE FOR THE BAHÍA DE CARÁQUEZ TOURIST DESTINATION.
}

\begin{abstract}
:
In the present investigation we analyzed the publicity in the social network of Facebook and its impact in the tourist development, case H Sports Café Bahía de Caráquez. The information obtained through the bibliographic field and the social network allowed a descriptive analysis through data tables in excel with the information of the publications and advertisements, taking into account the parameters of: views, likes, comments and number of times shared of the content. For the development of the analysis, Facebook was used as the main tool to gather information satisfactory for tourism development. It was verified that the visualizations of the published announcements in the Fanpage increased from 44639 in March of 2016 to 51362 in January of the 2017. he impact of ads per family of products representing cocktails was measured $40.10 \%$ (\$39629.96) of total sales, craft beers $25.31 \%$ (\$25010.43), wings, nachos and Nuggets 9.35\% (\$ 9239.64) and breakfasts 8.65\% (\$8549.45), showing that sales of the business have increased significantly. Therefore it is shown that advertising on Facebook social network contributes to the development of tourism and economic growth of business.
\end{abstract}

Keywords: advertising, tourism development, tourism, social network, sales

\section{PUBLICIDADE NAS REDES SOCIAIS COMO ALTERNATIVA SUSTENTÁVEL PARA O TURISTA BAY BAHÍA DE CARÁQUEZ - EQUADOR}

\section{Resumo:}

Na presente investigação analisamos a publicidade na rede social do Facebook e seu impacto no desenvolvimento turístico, caso H Sports Café de Caráquez. As informações obtidas através do campo bibliográfica e a rede social permitiu uma análise descritiva através de tabelas de dados no Excel Com as informações das publicações e propagandas, tendo em conta os parâmetros de: opiniões, gostos, comentários e número de vezes compartilhados do conteúdo. Para o desenvolvimento da análise, o Facebook foi utilizado como a principal ferramenta para coletar informações para o desenvolvimento do turismo. Verificou-se que as visualizações dos anúncios publicados na Fanpage aumentou de 44639 em Março de 2016 para 51.362 em janeiro de 2017. I do impacto de anúncios por família de produtos representativos Medido $40.10 \%$ foi cocktails $(\$ 39.629,96)$ de todos os sais, cervejas $25,31 \%$ de artesanato $(\$ 25.010,43$ ), asas, nacho e Nuggets $9,35 \%$ ( $\$ 9239,64)$ e pequenos-almoços $8,65 \%$ (\$ 8.549,45), mostrando sais do negócio que tem aumentado significativamente. Por isso, é mostrado que a publicidade na rede social Facebook contribui para o desenvolvimento do turismo e crescimento econômico dos negócios.

Palavras chave: publicidade, desenvolvimento do turismo, turismo, rede social, vendas 


\section{INTRODUCCIÓN:}

La publicidad ha sido desde su nacimiento un imán del talento que ha permitido a las empresas y negocios posicionarse en el mercado cada vez más competitivo. La hermana rica de la comunicación, ha contado con todos los recursos económicos, tecnológicos y profesionales a su alcance para el diseño y la elaboración del mensaje; un mensaje al servicio de las marcas. Informar y seducir, convencer con el objetivo final de vender un producto, un "posicionamiento" o una idea. Un proceso de creación sofisticado y complejo en el que hasta hace muy poco tiempo tan solo participaban anunciante y agencia, y los medios de comunicación, el vehículo para llegar a las diferentes audiencias y la pareja de hecho de la publicidad durante décadas. (Solana, 2011)

En el Ecuador, la primera imprenta fue traída desde España por la Compañía de Jesús, en el año de 1750, la misma que en un principio estuvo destinada de forma exclusiva para la publicación de obras religiosas. Posteriormente, se crearon varios periódicos que constituyeron los primeros soportes para los anuncios publicitarios. De esta forma, el desarrollo continuo de dicha industria, sumado a la aparición de la radio a finales del siglo XX, conllevó a un consecuente progreso de la actividad publicitaria. Es así que, el 22 de mayo de 1968 se crea la Asociación Ecuatoriana de Agencias de Publicidad (AEAP), cuyos objetivos principales eran profesionalizar la actividad publicitaria y generar una relación sólida con los medios de comunicación y anunciantes, en beneficio de los consumidores. (Saritama, 2015)

Pocas son las fuentes bibliográficas que se encuentran en el Ecuador sobre el origen yel desarrollo de la publicidad, tanto para estudiantes como profesionales el acceso a dicha información es casi nula, lo que limita el conocimiento y al mismo tiempo crea unafalta de aprecio por esta apasionante profesión.

En Bahía de Caráquez existen una gran variedad de cafeterías, bares y restaurantes considerados como una opción de oferta turística, los mismos que cuentan con una variedad de productos para ofrecer a los turistas y visitantes, además cuentan con un ambiente diferente y único capaz de brindar una experiencia agradable y diferente por el cual se caracteriza la ciudad.
Gráfico 1.

Descripción de cantidad de usuarios de Facebook en Ecuador

\begin{tabular}{l|c|c}
\hline \multicolumn{1}{c|}{ Ciudad } & Usuarios(miles) & $\%$ \\
\hline Guayaquil & $2,700.00$ & $30.34 \%$ \\
\hline Quito & $2,400.00$ & $26.97 \%$ \\
\hline Ambato & 410.00 & $4.61 \%$ \\
\hline Cuenca & 390.00 & $4.38 \%$ \\
\hline Portoviejo & 270.00 & $3.03 \%$ \\
\hline Loja & 170.00 & $1.91 \%$ \\
\hline Machala & 140.00 & $1.57 \%$ \\
\hline Esmeraldas & 120.00 & $1.35 \%$ \\
\hline Ibarra & 93.00 & $1.04 \%$ \\
\hline Otros & $2,207.00$ & $24.80 \%$ \\
\hline Total & $8,900.00$ & $100.00 \%$ \\
\hline .bo & & \\
\hline
\end{tabular}

Fuente:http://blog.formaciongerencial.com/2016/02/01/estadistica sfacebookecuador/

Analizando la cantidad de usuarios de Facebook en las principales ciudades del Ecuador, se tiene como principal Guayaquil con la mayor cantidad de usuarios seguida por Quito, Cuenca y Portoviejo, pudiendo ser estas ciudades visitantes que fomenten el desarrollo turístico de la ciudad de Bahía de Caráquez.

Sin embargo las cafeterías, bares y restaurantes no utilizan adecuadamente las redes sociales como Facebook para promover el turismo de la ciudad siendo esta la red social con más seguidores en el Ecuador y no tiene competencia, motivo por el cual constituye una herramienta para estar en contacto y mantener informados a sus públicos objetivos y potenciales de los productos y servicios que ofrecen y promover y desarrollar el turismo de la ciudad. El problema del negocio es la carencia de publicidad eficiente y de diseño que incida de forma positiva en las ventas. 
Frank Ángel Lemoine Quintero - Gema Lourdes Lopéz Zambrano - Wilfredo Valls Figueroa

La presente investigación tiene como objetivo analizar la publicidad de $\mathrm{H}$ Sports Café y su impacto en el desarrollo turístico en la ciudad de Bahía de Caráquez, para tener una noción clara de esta herramienta y su importancia para el desarrollo del turismo. Por lo que se hará un estudio comparativo a través de la red social de Facebook mediante los parámetros de: personas alcanzadas, likes, comentarios y el número deveces compartido de cada anuncio o publicación del antiguo y el actual administrador. Se medirá también los niveles de impactos de los anuncios por familia de productos, para verificar que está generando un aumento significativo en las ventas del negocio.

Se llega a justificar el presente estudio puesto que según (Ponce, 2016), Más de 50 millones de empresas utilizan Facebook como canal de negocios y Ecuador no es la excepción. A la fecha de esta publicación, Ecuador cuenta con una audiencia de 8'900.000 de usuarios, de los cuales 1'400.000 son administradores de páginas Facebook, muchas de las cuales pertenecen a marcas, emprendimientos e intereses comunes de grupos de usuarios.

Esto significa que la utilización de Facebook como una herramienta para comunicar o hacer publicidad por parte de las empresas está cada día en constante crecimiento, pues este medio permite llegar a personas o clientes reales y potenciales que se encuentran a 50 metros del negocio, siendo este factor muy importante para la difusión de información y anuncios.

\section{MARCO REFERENCIAL:}

Se conoce como Publicidad a aquella técnica destinada a difundir o informar al público sobre un bien o servicio a través de los medios de comunicación (televisión, cine, radio, revistas, Internet) con el objetivo de motivar al público hacia una determinada acción de consumo. La publicidad informará al consumidor potencial de un bien o servicio acerca de los beneficios que presenta este y resaltará las diferencias que lo distinguen de otras marcas. $(A B C)$

Según la American Marketing Asociation, la publicidad consiste en "la colocación de avisos y mensajes persuasivos, en tiempo o espacio, comprado en cualesquiera de los medios de comunicación por empresas lucrativas, organizaciones no lucrativas, agencias del estado y los individuos que intentan informar y/o persuadir a los miembros de un mercado meta en particular 0 a audiencias acerca de sus productos, servicios, organizaciones o ideas" (Thompson, brd.unid.edu.mx, 2005)

Para Stanton, Walker y Etzel, autores del libro "Fundamentos de Marketing", la publicidad es "una comunicación no personal, pagada por un patrocinador claramente identificado, que promueve ideas, organizaciones o productos. Los puntos de venta más habituales para los anuncios son los medios de transmisión por televisión y radio y los impresos (diarios y revistas). Sin embargo, hay muchos otros medios publicitarios, desde los espectaculares a las playeras impresas y, en fechas más recientes, el internet" (Thompson, brd.unid.edu.mx, 2005)

La publicidad en la era digital para ser entendida en su base, se tiene que entender que se debe al desarrollo de la computación, la programación, los dispositivos móviles, y sobre todo el internet. (usr.uvic, 2012)

La aparición de la tecnología ha ahecho cambiar todo el esquema de la comunicación, y así la mentalidad de la gente. En cuanto a la publicidad, las plataformas digitales se han convertido en un peso relevante en todas las campañas publicitarias. (usr.uvic, 2012)

Según (Cerezo, 2012) en su libro la publicidad en la era digital otro aspecto que influye la tecnología en la publicidad es a la hora de la elaboración de los mensajes, que son mejorados por los entornos que se plantean, mejorando la estética, aplicando nociones de libertad para interactuar, y que a su vez sirvan de base para que los mismos receptores puedan crearse nuevos significados y a su vez lo experimenten con sensaciones que hagan implicarse.

En la actualidad la publicidad en los medios tradicionales sigue teniendo relevancia en el medio, sin embargo es muy notorio como la publicidad en la era digital ha tenido su auge haciendo cambiar las estrategias de difusión y comunicación de muchas empresas y negocios, además de que permite estar en contacto con el cliente e incentivar a la compra o consumo del producto, es el medio publicitario más económico y permite tener un alto índice en la notoriedad y engagement con la marca.

Facebook es un fenómeno que ha cambiado la forma de hacer marketing y publicidad, permitiendo que las empresas conozcan fácilmente los gustos de los clientes y se conecten directamente con ellos. (Franco, 2009) 
La publicidad ha revolucionado con las nuevas tecnologías y la internet, en la actualidad las redes sociales permiten llegar a una gran cantidad de personas de una forma más rápida y eficaz, siendo esta herramienta el mayor exponente de marketing viral, porende las empresas no han desaprovechado la oportunidad y han apostado por este medio, el mismo que a través de una Fanpage les permite describir quienes son, que ofrecen, mostrar sus productos y anunciar sus páginas webs y demás redes sociales.

Bajo un criterio estadístico, "Turismo es el conjunto de las relaciones y fenómenos producidos por el desplazamiento y permanencia de personas fuera de su lugar de domicilio, en tanto, dicho desplazamiento y permanencia no estén motivados por una actividad lucrativa" (Naciones, 1937).

Para la (Organización Mundial del Turismo, 2015), el turismo comprende las actividades que realizan las personas durante sus viajes y estancias en lugares distintos al de su residencia habitual por menos de un año y con fines de ocio, negocios, estudio, entre otros.

Mientras para (Walter Hunziker y Kart Krapf, 1942), Turismo es el conjunto de relaciones y fenómenos que se producen como consecuencia del desplazamiento y estancia temporal de personas fuera de su lugar de residencia, siempre que no esté motivado por razones lucrativas.

Cabe resaltar que los usuarios a la hora de planificar un viaje, elegir destinos turísticos e incluso a la hora de decidirse entre un alojamiento $u$ otro, utilizan las redes sociales para para disponer de la opinión de otros usuarios sobre los destinos potenciales, los servicios que pueden encontrar allí como: hoteles, restaurantes, cafeterías, bares u otros.

Por esta importante razón las recomendaciones de otros usuarios son de vital importancia en el sector turístico y su desarrollo, puesto que cada año millones de turistas y viajeros planifican sus viajes y visitas a los diferentes destinos turísticos.

Son muchos los viajeros que utilizan el internet durante su viaje para gestionar o dar a conocer las actividades que realizan en el destino. Por medio de las redes sociales mantienen un dialogo directo haciendo preguntas de lugares atractivos y especiales, siendo la repuesta de la gente crucial para generar turismo.

Además a través de los dispositivos móviles con conexión a internet, los usuarios se dan un tiempo para compartir la experiencia con sus amigos, familiares 0 sus diferentes contactos de las redes sociales y difundir sus recomendaciones, siendo este acto lo más importante e interesante de una buena experiencia y servicio.

\section{METODOLOGÍA:}

La metodología utilizada para la elaboración del presente estudio científico consistió en un análisis de la publicidad en Facebook, desde una fundamentación teórica que fundamenta desde el estado del arte a la concepción descriptiva con el objetivo de analizar la publicidad y su impacto en el desarrollo turístico caso $\mathrm{H}$ Sports Café Bahía de Caráquez, para tener noción del número de visualizaciones, likes, comentarios y número de veces compartida de los anuncios publicado en esta red social entre el antiguo y el actual administrador del Fanpage y poder hacer un comparación con las ventas de los productos y constatar que estos están teniendo un impacto significativo en las ventas del negocio. Además se realizó un análisis comparativo a través de los anuncios por familia de productos y si durante este periodo las ventas tienen tendencia a la alza 0 a la baja, el trabajo de campo se efectuó en un mes empezando el 1 de enero y culminando el 1 de febrero del 2017.

Desde la fundamentación práctica se consideró un total de 4 meses que comprenden desde diciembre del año 2015 a marzo del 2016, en el que las publicaciones y anuncios fueron realizadas por el antiguo administrador y desde octubre del 2016 a febrero del 2017 periodo en el que el Fanpage empezó a tener un nuevo administrador. La tabulación y análisis de los datos e información recolectada se lo realizo en Excel, utilizando técnicas de análisis, para una mejor redacción y comprensión de los resultados.

\section{RESULTADOS:}

Se realiza un estudio histórico estadístico teniendo en cuenta 4 parámetros evaluativos con la finalidad de medir un comportamiento de las ventas y medir la efectividad de las publicidad realizada en el negocio para realizar una correlación de rentabilidad e impacto del uso de los medio publicitarios, para este estudio se escogerá la red social de Facebook por ser la de mayor nivel de difusión y de aceptación por la población o la zona costa de Manabí. 
Frank Ángel Lemoine Quintero - Gema Lourdes Lopéz Zambrano - Wilfredo Valls Figueroa

Primeramente se hará un análisis de la primera dirección administrativa de la red para conocer la efectividad escogiendo los meses de diciembre 2015 hasta marzo 2016, tal como se muestra en la siguiente tabla 1.

\section{Tabla № 1.}

Datos de las publicaciones del antiguo administrador

\begin{tabular}{|l|l|l|l|l|}
\hline Parámetros & Dic. & Ene. & Feb. & Mar. \\
\hline Visualizaciones & 13235 & 19968 & 32429 & 44639 \\
\hline Likes & 467 & 1013 & 1034 & 1368 \\
\hline Comentarios & 18 & 40 & 35 & 99 \\
\hline Compartir & 63 & 45 & 100 & 149 \\
\hline Total & 13783 & 21066 & 33598 & 46255 \\
\hline
\end{tabular}

Fuente: Elaboración propia.
Según los datos recopilados y obtenidos de Facebook se puede decir que el antiguo administrador obtuvo 13235 visualizaciones en diciembre, 19968 en enero, 32429 en febrero y 44639 en marzo, lo que significa que si hubo una gran cantidad de usuarios que accedieron a la página y se interesaron por las publicaciones y su contenido.

En lo que corresponde a likes obtuvo un total de 467 en diciembre, 1013 en enero, 1034 en febrero y 1368 en marzo. Asimismo generó en sus publicaciones 18 comentarios en el mes de diciembre, 40en el mes de enero, 35 en febrero y 99.Y finalmente los anuncios compartidos en la página fueron compartidos 63 veces en el mes de diciembre, 45 en el mes de enero, 100 en febrero y 149 veces en el mes de marzo como se refleja en el siguiente gráfico.

\section{Gráfico 2: Resultados estadístico de diciembre a marzo 2016}

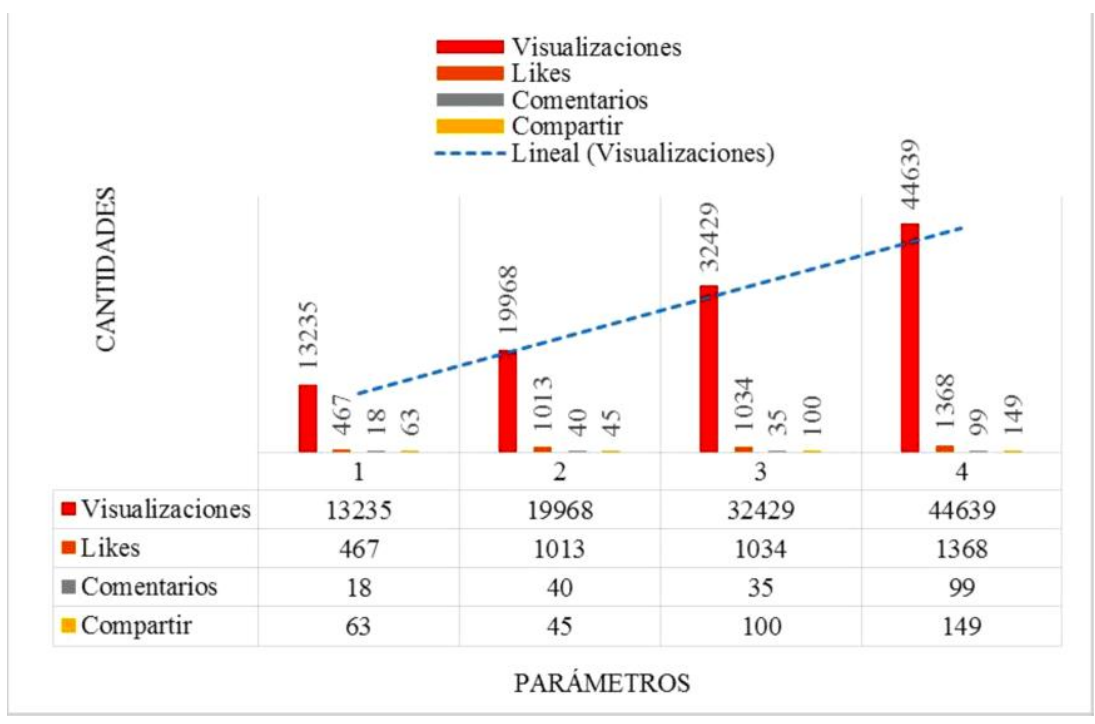

Fuente: Elaboración propia 
Se realiza un estudio midiendo los resultados de las ventas de octubre 2016 a Enero 2017 para analizar el comportamiento de las mismas tendiendo presente los mismos parámetros a medir.

De acuerdo con los datos obtenidos de Facebook, se puede asegurar que el actual administrador obtuvo un total de 12583 visualizaciones en octubre, 13428 noviembre, 49484 diciembre y 51362 en enero, lo que se significa el contenido y los anuncios publicado fueron vistos una cantidad representativa de usuarios, los mismo que se interesaron por estas publicaciones sin necesidad de haber interactuado o ingresado a la página según se muestra en la tabla 2.

Tabla № 2. Datos de las publicaciones del actual administrador

\begin{tabular}{r|c|c|c|c}
\hline Parámetros & Octubre & Noviembre & Diciembre & Enero \\
& & & & \\
\hline Visualizaciones & 12583 & 13428 & 49484 & 51362 \\
\hline Likes & 233 & 307 & 1174 & 680 \\
\hline Comentarios & 11 & 11 & 14 & 59 \\
\hline Compartir & 43 & 43 & 116 & 116 \\
\hline Total & 12870 & 13789 & 50788 & 52217 \\
\hline
\end{tabular}

Fuente: Elaboración Propia
En lo que pertenece a likes obtuvo un total de 233 en octubre, 307 en noviembre, 1174 en diciembre y 680 en enero, pudiendo decir que una cantidad importante de usuarios si les gustaron las publicaciones. Además se generó en sus publicidades 11 comentarios en el mes de octubre, 11 en el mes de noviembre, 14 en diciembre y 59 en enero.Finalmente las publicaciones compartidas en la página fueron en un total de 43 veces en el mes de octubre, 43 en el mes de noviembre, 116 en diciembre y 116 veces en el mes de marzo, deduciendo que el contenido fue agradable para losseguidores y fans de la página y por ende fueron compartidos en una gran cantidad de veces, gráfico 3 .

\section{Gráfico 3. Estudio comparativo de los meses octubre a}

enero

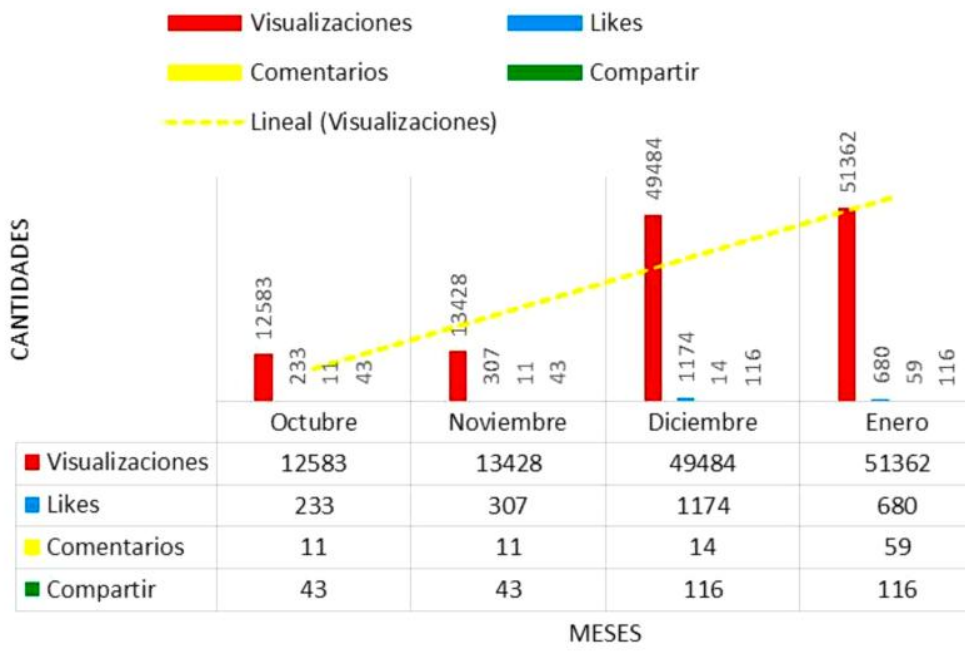

En lo que corresponde al análisis por familia de productos y su relación con las ventas en el período de Febrero del 2016 a febrero del 2017, la familia de productos que más genero ventas es la de cocteles con un importe total de $\$ 39629,96$ aproximadamente representando el $40,10 \%$ del total de las ventas de todo un año. Le siguen las familias de: cervezas artesanales con un importe de $\$ 25010,43$ aproximados representando el $25,31 \%$, asimismo las alitas, nachos y Nuggets con un importe de 9239, 64 aproximados siendo este el $9,35 \%$ y continuando con los desayunos que obtuvieron un importe $\$ 8549,45$ aproximados 
representando el $8,65 \%$ de las ventas totales del negocio.

Se tomaron en cuenta los porcentajes más relevantes y que más ventas generan al negocio, no descartando que las demás familias de productos como: los Sánduches 3,29\%, hamburguesas 2,85\%, jugos 2,78\%, aguas $0,57 \%$, coffes $2,44 \%$, Smoothies $0,92 \%$, otras bebidas $2,08 \%$, postres $1,03 \%$, ensaladas $0,58 \%$ y camisas $0,05 \%$ que también constituyen un porcentaje significativo en las ventas del negocio, tabla 3.

\section{Tabla № 3 . Familia de productos}

\begin{tabular}{|l|l|l|}
\hline Familia de Productos & Importe & $\%$ \\
\hline Alitas, Nachos y Nuggets & 9239,64 & $9,35 \%$ \\
\hline Cocteles & 39629,96 & $40,10 \%$ \\
\hline Desayunos & 8549,45 & $8,65 \%$ \\
\hline Hamburguesas & 2817,06 & $2,85 \%$ \\
\hline Coffes & 2411,74 & $2,44 \%$ \\
\hline Jugos & 2742,98 & $2,78 \%$ \\
\hline Aguas & 562,60 & $0,57 \%$ \\
\hline Postres & 1019,02 & $1,03 \%$ \\
\hline Otras Bebidas & 2054,93 & $2,08 \%$ \\
\hline Sánduches & 3253,28 & $3,29 \%$ \\
\hline Smoothies & 909,58 & $0,92 \%$ \\
\hline Cerveza Artesanal & 25010,43 & $25,31 \%$ \\
\hline Ensaladas & 574,81 & $0,58 \%$ \\
\hline Totalisas & 98820,48 & $100 \%$ \\
\hline & & \\
\hline
\end{tabular}

En que corresponde a las ventas en los meses de octubre del 2016 a febrero del 2017 las ventas han tenido el siguiente comportamiento: en octubre se obtuvo un total de $\$ 15746,88$ aproximados, en noviembre $\$ 7844,36$ mostrando una baja, en diciembre siguieron bajando obteniendo un total de $\$ 5526,71$, en enero empezaron a aumentar resultando $\$ 6084,78$ de ventas, y en el mes de febrero las ventas se dispararon significativamente llegando a obtener un total de $\$ 27041,00$ en solo un mes superando al mes de octubre con una diferencia muy importante y notoria de $\$ 11295,00$,

A pesar de que en los primeros meses del año las ventas en la mayoría de los negocios suelen ser un poco bajas a $\mathrm{H}$ Sports Café le fue muy bien en el rendimiento de sus ventas, por lo que se puede deducir que las ventas tienen una tendencia al alza según se muestra en el gráfico 5.

\section{Gráfico $\mathbf{N}^{\circ} 5$}

\section{ventas de Octubre de 2016 a Febrero 2017}

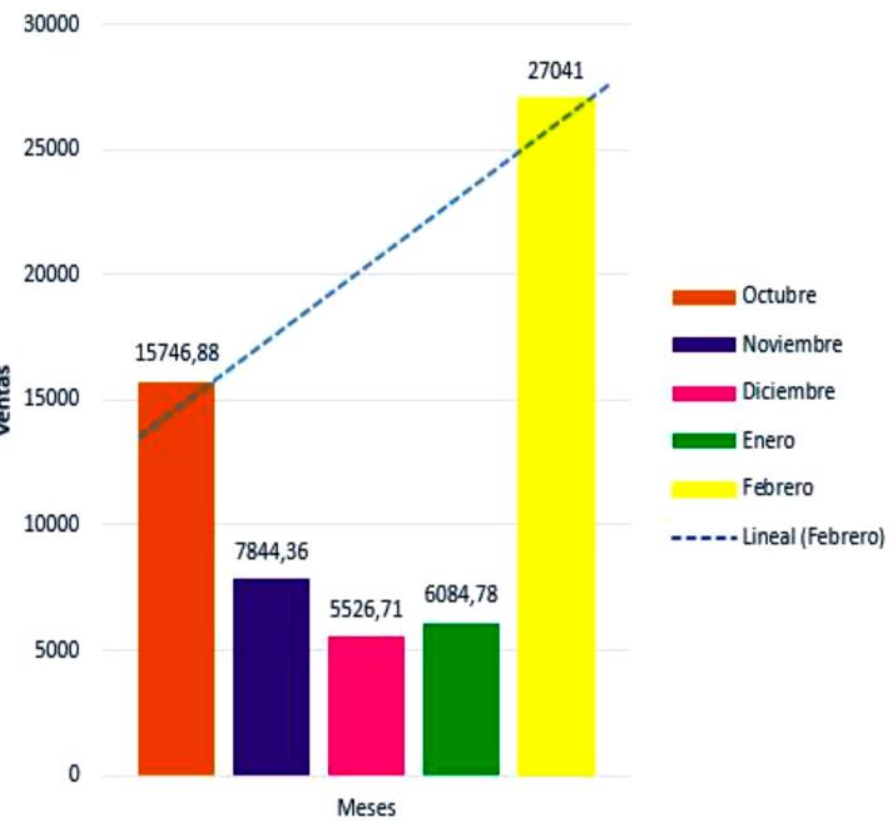

Fuente: Elaboración Propia 
Es elemental para lograr impacto positivos en las redes sociales analizar la disponibilidad de sistemas de cómputos y de tecnologías que permitan el estudio del uso de estos en los clientes como factor de impacto y reconocimiento de una probabilidad del uso de las redes sociales en el cantón.

El software REDATAM es una solución tecnológica desarrollada por el Centro Latinoamericano y Caribeño de Demografía (CELADE), División de Población de la Comisión Económica para América Latina y el Caribe (CEPAL), de las Naciones Unidas (UN), con el propósito de aportar un conjunto de herramientas a los países de la región, para la caracterización y el análisis local, provincial y regional de los microdatos censales, a partir del procesamiento de los datos en línea, con la posibilidad de obtener tablas, gráficos y/o mapas, además permite el estudio estadístico poblacional del Ecuador que a su vez contiene todas las variables investigadas a nivel de vivienda, exceptuando las consideradas confidenciales, como la dirección domiciliaria y algunas variables catastrales.

A través del software el cual se trabajó mediante variables cruzadas, para determinar cuántas personas poseen computadoras en el Cantón, esto se hizo con la finalidad de fijar los rangos de edad más representativos para la investigación que se está realizando; los datos obtenidos fueron los siguientes:
De acuerdo a los datos de la tabla 4 , se puede determinar que el $80 \%$ de la población que representa a 5.207 habitantes de entre 1 a 49 años si disponen de una computadora, debido a que estos rangos de edad están entre los niveles más representativos para nuestra investigación, se puede decir que es factible hacer publicidad dirigida a ellos porque poseen este instrumento para recibir los anuncios, por lo tanto esto ayuda a impulsar el desarrollo del turismo en la Ciudad; el $20 \%$ que representa a 1.274 habitantes de entre 50 a 100 años también poseen computadoras, pero forman parte de los niveles menos representativos para la investigación que se está realizando. Estos porcentajes representan el $11 \%$ de la población total, lo cual quiere decir 6.481 personas del Cantón Sucre.

De acuerdo a los datos de la tabla, también se refleja que el $81 \%$ de la población que representa a 40.627 personas de entre 1 a 49 años, no dispone de una computadora, y el otro $19 \%$ que representa a 9.617 habitantes de entre 50 a 100 años tampoco disponen dela misma. Esto quiere decir según datos globales de la población, que el $89 \%$ de residentes del Cantón que representa a 50.244 personas, carecen de una computadora. Con la ayuda del software también se pudo establecer mediante variables cruzadas, la cantidad de viviendas que disponen de computadoras según la zona a la que pertenecen, sea esta urbana o rural, los datos obtenidos fueron los que se muestran a continuación en la tabla5.

Tabla 5: Disponibilidad de computadoras por viviendas área urbana y rural

\begin{tabular}{c|r|r|r|r|r|r}
\hline Área Urbana o Rural & \multicolumn{6}{|l}{ Dispone de computadora } \\
\hline & \multicolumn{1}{|l|}{ Si } & \multicolumn{1}{|l}{ No } & Total & si & ro & lotal \\
\hline 1. Área Urbana & 1180 & 4356 & 5536 & $21 \%$ & $79 \%$ & $100 \%$ \\
\hline 2. Área Rural & 478 & 9326 & 9804 & $5 \%$ & $95 \%$ & $100 \%$ \\
\hline Total & 1658 & 13682 & 15340 & & & \\
\hline
\end{tabular}

Fuente: Programa de datos estadísticos REDATAM

De acuerdo a los datos de la tabla se puede determinar que, en la zona urbana 1,180 viviendas sí disponen de computadoras, esto representa el $21 \%$ de viviendas en esta área, mientras que 4,356 viviendas que representan el $79 \%$ de domicilios totales en la zona rural no poseen servicios de cómputo. 
En el área rural, 478 casas sí disponen de computadoras, representando el $5 \%$ de viviendas de esta área, mientras que 9,326 domicilios que representan el $95 \%$ de casas totales carecen de computadoras.

\section{Análisis del servicio de telefonía celular}

El estudio basado en las fuentes estadísticas del software REDATAM permitió trabajar con dos variables cruzadas las cuales fueron grupo de edades y número de teléfonos a nivel de persona con la finalidad de determinar los segmentos más representativos con esta tecnología, cuyos resultados fueron los siguientes:

Tabla 6: Disponibilidad de teléfono celular en el cantón Sucre por rangos de edad

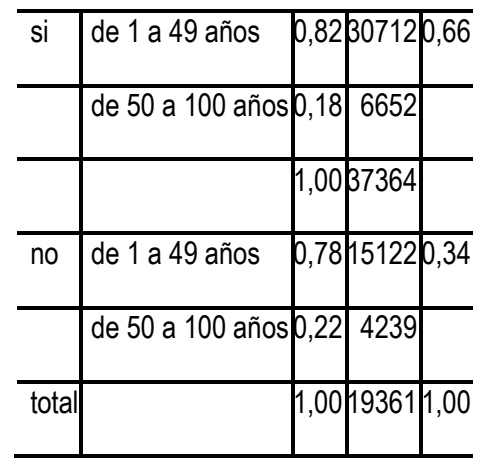

Fuente: Programa de datos estadísticos REDATAM

De acuerdo a los datos de la tabla, se puede determinar que el $82 \%$ de la población que representa a 30.712 habitantes de entre 1 a 49 años si disponen de un teléfono celular, debido a que estos rangos de edad están entre los niveles más representativos para nuestra investigación, se puede decir que es factible hacer publicidad dirigida a ellos, principalmente porque representan un número elevado de habitantes que puede recibir la información y difundirla, brindando de esta manera un aporte al turismo de la Ciudad; el 18\% que representa a 6.652 habitantes de entre 50 a 100 años también poseen celulares, pero forman parte los niveles menos representativos para la investigación que se está realizando. Estos porcentajes representan el $66 \%$ de la población total, lo cual quiere decir 37.364 personas del Cantón Sucre.
De acuerdo a los datos de la tabla, también se refleja que el $78 \%$ de la población que representa a 15.122 personas de entre 1 a 49 años, no poseen un teléfono celular, y el restante $22 \%$ que representa a 4.239 habitantes de entre 50 a 100 años tampoco disponen del mismo. Esto quiere decir según datos globales de la población, que el $34 \%$ de residentes del Cantón que representa a 19.361 personas, no disponen de un teléfono celular.

El software de bases estadísticas REDATAM, también permitió investigar mediante el uso de variables cruzadas, la cantidad de viviendas que poseen teléfono celular por las zonas a las que pertenecen, sean estas urbana o rural. Los resultados obtenidos fueron los representados en la tabla 7.

Tabla 7: Disponibilidad de computadoras por viviendas área urbana y rural

\begin{tabular}{c|c|c|c|c|c|c}
\hline $\begin{array}{c}\text { Área Urbana } \\
\text { o Rural }\end{array}$ & \multicolumn{5}{|c}{ Disponibilidad de teléfono celular } \\
\hline & & N & Total & i & n & total \\
& & & & & & \\
\hline 1. Área Urbana & 4049 & 1487 & 5536 & $73 \%$ & $27 \%$ & $100 \%$ \\
\hline 2. Área Rural & 5615 & 4189 & 9804 & $57 \%$ & $43 \%$ & $100 \%$ \\
\hline Total & 9664 & 5676 & 15340 & & & \\
\hline
\end{tabular}

Fuente: Programa de datos estadísticos REDATAM

De acuerdo a los datos de la tabla se puede determinar que en la zona urbana, 4,049 viviendas si poseen teléfonos celulares, representando el $73 \%$ de viviendas en esta zona, mientras que 1,487 domicilios que representan el $27 \%$ de viviendas totales de esta áreano poseen teléfonos celulares.En la zona rural 5,615 casas, que representan el $57 \%$ de domicilios en esta área si poseen un teléfono, mientras que 4,189 viviendas que representan el $43 \%$ restante de hogares no disponen de un teléfono celular.

Desarrollar una propuesta dirigida al diseño de una campaña de publicidad en redes sociales con la finalidad de promocionar al Cantón como destino turístico, no solo a nivel provincial, sino también nacional, resaltando los principales sitios turísticos, así como su gastronomía, culturas, hospitalidad y riqueza 
natural. Por medio de esta lograr mayor reconocimiento de los atractivos turístico, y por ende obtener mejor posicionamiento de las empresas de servicios hoteleros, fomentando al crecimiento empresarial y aportando al crecimiento económico y social.

\section{CONCLUSIONES:}

Se realizó un estudio del estado del arte que aportara al marco teórico de la investigación y a la fundamentación científica del objetivo de estudio en el que se logró obtener una noción clara y precisa de la importancia de Facebook como herramienta publicitaria de redes sociales con mayor número de usuarios según estadísticas registradas y por ende como un medio de promoción y desarrollo del turismo sostenible a nivel local, regional y nacional.

Se realizó un análisis comparativo de la publicidad mediante los parámetros de personas alcanzadas, likes, comentarios y el número de veces compartido de cada anuncio o publicación del antiguo y el actual administrador, obteniendo como resultando un aumento del número de personas a las que llega las publicaciones en relación con el antiguo administrador de la Fanpage que se representa en el número de clientes atendidos según las estadísticas de ventas.

Se midió correctamente el impacto de los anuncios en las ventas del negocio por medio de familias de productos, verificando que cada una de ellas genera un importe muy significativo en la permanencia del negocio en el mercado, destacando de esta forma que la publicidad permite obtener resultados satisfactorios para las ventas y el desarrollo turístico sostenible en la zona objeto estudio.

Además se analizaron las ventas en relación con la publicidad, resultando que las mismas si han tenido un crecimiento positivo, demostrando de esta forma que Facebook es un herramienta publicitaria eficaz, permite generar ingresos y a la misma vez informar a sus clientes-seguidores que visiten el negocio que es parte de un destino turístico de la ciudad de Bahía de Caráquez.

\section{REFERENCIAS:}
ABC,D.(s.f.).Definición ABC.Obtenido de Definición ABC:http://www.definicionabc.com/comunicacion/ publicidad.php

Díaz, D. E. (9 de Agosto de 2008). Darío Ezequiel Díaz.blogspost.com. Obtenido de Darío Ezequiel Díaz.blogspost.com: http://darioezequieldiaz.blogspot.com/200 8/08/desarrollo-economico-primeraparte.html

Franco, C. (18 de Mayo de 2009). bligoo. Obtenido de bligoo: http://manuelgross.bligoo.com/content/vie w/516215/El-uso-de-Facebook-comoherramienta-demarketing.html\#.WLe61DuGPIU

Gómez, J. C. (28 de Septiembre de 2008). Gestiopolis. Obtenido de Gestiopolis: http://www.gestiopolis.com/teoria-yconceptos-de-sunkel-y-paz-sobredesarrollo-economico/

MindTools.(s.f.). MindTools.com. Obtenido de https://www.mindtools.com/pages/article/new $\underline{\text { TMC 09.htm }}$

Ministeriode Turismo. (2015). Turismo

en Cifra: Boletines Estadisticos. Quito. Recuperado el 22 de Julio de 2016, de http://repositorio.ucsg.edu.ec/bitstream/3317/496 4/1/T-UCSG-PRE-ESP-AETH-265.pdf

$\begin{array}{lll}\text { Naciones, } & \text { S. d. } & \text { (1937). } \\ & \text { SlideShare. } & \text { Obtenido }\end{array}$ deSlideShare:http://es.slideshare.net /fatimacordova/definiciones-de-turismodiferentes-s-autores-diferente

Organización Mundial del Turismo. (2015). Turismo.

Ponce, J. P. $(01$ de Febrero de 2016). Formaciongerencial.

Obtenido de Formaciongerencial: http://blog.formaciongerencial.com/2016/02/01/est adisticasfacebookecuador/ 